\title{
REFLEXÕES SOBRE QUESTÕES CONTEMPORÂNEAS EMERGENTES: ACESSIBILIDADE DIGITAL NA REMAT - REVISTA ELETRÔNICA DA MATEMÁTICA
}

Greice da Silva Lorenzzetti Andreis ${ }^{1}$; Luís Henrique Ribeiro da Silva²; Rodrigo Sychocki da Silva $^{3}$; Katia Arcaro ${ }^{4}$; Henri Luiz Fuchs ${ }^{5}$; David Matos Milhomens ${ }^{6}$

\section{Como citar:}

ANDREIS, G. S. L.; SILVA, L. H. R.; SILVA, R. S.; ARCARO, K.; FUCHS, H. L.; MILHOMENS, D. M. Reflexões sobre questões contemporâneas emergentes: acessibilidade digital na remat - revista eletrônica da matemática. In: ABEC MEETING, 1, 2017, Curitiba. Anais... Curitiba: Associação Brasileira de Editores Científicos, 2017. p. 6-13.

http://dx.doi.org/10.21452/abecmeeting.2017.002

Resumo: A REMAT: Revista Eletrônica da Matemática (e-ISSN 2447-2689) ${ }^{1}$ tem como missão compartilhar práticas educativas e resultados de pesquisas que se relacionam com a Matemática. Em busca da consolidação da revista perante a comunidade acadêmica, a Equipe Editorial da REMAT vem trabalhando no aprimoramento dos processos editoriais, estejam eles relacionados à qualificação do que é produzido ou à acessibilidade digital. A REMAT, editada e publicada pelo Instituto Federal de Educação, Ciência e Tecnologia do Rio Grande do Sul (IFRS) em seu Portal de Periódicos ${ }^{2}$, é um periódico científico eletrônico de acesso livre, com publicação semestral, que visa publicizar produções originais de pesquisadores, sendo estas organizadas em três seções: Matemática em Contextos Técnicos e/ou Tecnológicos, Ensino de Matemática e Matemática Pura e/ou Aplicada. Este artigo apresenta um panorama das reflexões e ações já desenvolvidas na REMAT, incluindo-se o aperfeiçoamento rumo à acessibilidade digital.

Palavras-chave: REMAT. Acessibilidade Digital. Processos Editoriais. Matemática.

Abstract: REMAT: Revista Eletrônica da Matemática (e-ISSN 2447-2689) has as mission to share educatives practices and research results that relate with the Math. Looking for the journal consolidation in front of the academic community, REMAT Editorial Staff is working in the editorials processes improvement, whether linked to the qualification of the produced material or linked to the digital accessibility. REMAT, edited and published by Instituto Federal de Educação, Ciência e Tecnologia do Rio Grande do Sul (IFRS) in its Portal of Scientific Journals, is an electronic scientific journal that offers free access, with semi-annual publication, and aims to publish researchers original productions, being these organized in three sections: Mathematics in Technical and/or Technological Contexts, Mathematics Teaching and Pure and/or Applied Mathematics. This article presents a panorama of reflections and actions already developed in REMAT, including the enhancement towards the digital accessibility.

Keywords: REMAT. Digital Accessibility. Publishing Processes. Mathematics.

\section{HISTÓRICO}

A Revista Eletrônica da Matemática (REMAT) teve início em 2015, idealizada por um grupo de docentes do Instituto Federal de Educação, Ciência e Tecnologia do Rio Grande do Sul (IFRS), do Campus Caxias do Sul, com o objetivo de publicizar produções originais com enfoque no Ensino de Matemática, Matemática Aplicada e/ou Matemática Pura, bem como divulgar pesquisas sobre o uso de tecnologias digitais e suas contribuições para estes campos do conhecimento (AUTOR et al, ano 1). Foram publicadas duas edições em 2015. A primeira

\footnotetext{
${ }^{1}$ https://periodicos.ifrs.edu.br/index.php/REMAT/index.

2 https://periodicos.ifrs.edu.br/index.php/index.
} 
ANDREIS, G. S. L.; SILVA, L. H. R.; SILVA, R. S.; ARCARO, K.; FUCHS, H. L.; MILHOMENS, D. M.

"Reflexões sobre questões contemporâneas emergentes: acessibilidade digital na remat - revista eletrônica da matemática"

totalmente vinculada à Semana Acadêmica do Curso de Licenciatura em Matemática do IFRS, Campus Caxias do Sul, e a segunda com artigos provenientes de diferentes instituições de ensino.

Em 2016, a REMAT passou a apresentar uma seção especial, dedicada à Matemática desenvolvida nos Institutos Federais, tanto a nível de pesquisa científica quanto a nível de práticas de ensino executadas nos cursos técnicos, de graduação e de pós-graduação (AUTOR et al, ano 1). No mesmo ano, foi indexada junto ao Google Acadêmico e ao Diretório de Políticas Editoriais das Revistas Científicas Brasileiras (Diadorim). No final deste mesmo ano, as seções foram novamente reestruturadas, passando a ser: Matemática em Contextos Técnicos e/ou Tecnológicos, Ensino de Matemática e Matemática Pura e/ou Aplicada.

Além disso, ainda em 2016, com a finalidade de fomentar a qualificação do periódico, a Equipe Editorial foi reestruturada e ampliada, ficando composta por: Editor-chefe, Editor Adjunto, Editor Executivo, Comissão Editorial, Conselho Editorial Consultivo, Editores de Texto, Apoio Técnico e Avaliadores ad hoc (AUTOR et al, ano 2). A nova constituição do Conselho Editorial Consultivo e do grupo de Avaliadores ad hoc visou a atender a uma política de inclusão de pesquisadores de diferentes regiões e instituições do Brasil e do exterior. No início de 2017, o Apoio Técnico também passou a ter a contribuição de um bolsista que auxilia nos processos de gestão e de divulgação da revista.

Em janeiro de 2017, o periódico recebeu classificação no Qualis Periódicos, disponibilizada na Plataforma Sucupira da Coordenação de Aperfeiçoamento de Pessoal de Nível Superior (CAPES), em cinco áreas de avaliação:

- Ensino: B3

- Interdisciplinar: B5

- Matemática/Probabilidade e Estatística: B5

- Psicologia: B5

- Educação: C

A revista recebe submissões de artigos em fluxo contínuo, que são avaliados por avaliadores $a d$ hoc, de acordo com suas áreas de atuação, em um processo duplamente cego ${ }^{3}$. Os critérios de avaliação observam os seguintes aspectos: relevância e originalidade, clareza e pertinência dos objetivos, adequação do título ao conteúdo, estrutura coerente, clareza na metodologia, apresentação de resultados e discussão dos mesmos, citações e referências adequadas ao conteúdo.

\footnotetext{
${ }^{3}$ Considera-se "processo duplamente cego", toda avaliação de artigo da forma que o avaliador não conheça os metadados da submissão, nem saiba quem são os outros avaliadores do mesmo artigo, e que os autores também não saibam por quem seu artigo foi avaliado.
} 
ANDREIS, G. S. L.; SILVA, L. H. R.; SILVA, R. S.; ARCARO, K.; FUCHS, H. L.; MILHOMENS, D. M.

"Reflexões sobre questões contemporâneas emergentes: acessibilidade digital na remat - revista eletrônica da matemática" 
ANDREIS, G. S. L.; SILVA, L. H. R.; SILVA, R. S.; ARCARO, K.; FUCHS, H. L.; MILHOMENS, D. M.

"Reflexões sobre questões contemporâneas emergentes: acessibilidade digital na remat - revista eletrônica da matemática"

\section{AÇÕES DESENVOLVIDAS COM VISTAS À ACESSIBILIDADE DIGITAL}

A acessibilidade de um periódico em formato digital é um compromisso importante de ser assumido, demandando conscientização dos envolvidos nos processos editoriais, bem como disponibilidade de ferramentas adequadas no sistema de editoração. A busca pela acessibilidade digital tem sido um dos objetivos da REMAT desde sua criação e, nessa direção, pretende-se, para as edições futuras a partir de 2018, intensificar as ações relacionadas à acessibilidade digital.

Entende-se acessibilidade como uma possibilidade e condição de alcance para utilização, com segurança e autonomia, de espaços físicos, equipamentos ou informação por pessoa com deficiência ou com mobilidade reduzida (BRASIL, 2015). Outro conceito importante quando se fala de acessibilidade é o de inclusão que, conforme Sassaki (2009), é o processo pelo qual os sistemas comuns à sociedade são apropriados para toda a diversidade humana. Ainda, define-se barreira como um obstáculo que impede a participação de um indivíduo, ferindo seus direitos à acessibilidade, tal como a liberdade de movimento e o acesso à informação (BRASIL, 2015). Quando se fala em meio digital, no entanto, existem as barreiras tecnológicas que, conforme a Lei $\mathrm{n}^{\circ} 13.146$ (BRASIL, 2015), são aquelas que “[...] dificultam ou impedem o acesso da pessoa com deficiência às tecnologias”.

Algumas barreiras tecnológicas em um periódico eletrônico, que ferem o direito à acessibilidade, são percebidas por pessoas com deficiência visual. A deficiência visual "[...] pode ser entendida como a perda ou redução significativa da capacidade visual em ambos os olhos, mesmo após correção, tratamento clínico ou cirúrgico, resultando em cegueira ou baixa visão" (SALTON et a., 2017, p. 27). Com isso exposto, é necessário entender que os recursos utilizados por pessoas cegas e pessoas com baixa visão são distintos. Salton et al (2017) ressaltam que as pessoas cegas utilizam softwares que fazem a leitura da tela e a navegação por meio do teclado. Já pessoas que possuem baixa vilão utilizam, além de leitores de tela, ampliadores de tela e recursos de alto contraste (SALTON et al, 2017). No caso dos leitores de tela, é necessário que o documento disponibilizado nos meios eletrônicos esteja configurado de forma correta.

Nesse sentido, a REMAT tem disponibilizado um modelo editável no formato DOCX ${ }^{4}$ que possui estilos pré-configurados. Os estilos, além de promover maior organização da estrutura do texto, permitem que quando o documento for exportado para o formato PDF/A ${ }^{5}$

\footnotetext{
${ }^{4}$ DOCX é a extensão de arquivos suportados pelo editor de texto Microsoft Office Word.

${ }^{5}$ PDF/A é uma extensão de arquivos derivada do Portable Document Format (PDF), que torna o documento adequado para acesso e leitura durante um longo período de tempo.
} 
ANDREIS, G. S. L.; SILVA, L. H. R.; SILVA, R. S.; ARCARO, K.; FUCHS, H. L.; MILHOMENS, D. M.

"Reflexões sobre questões contemporâneas emergentes: acessibilidade digital na remat - revista eletrônica da matemática"

carregue as informações de forma a facilitar o uso de leitor de tela por pessoas cegas. O uso desse recurso, apoiando-se em Salton et al. (2017), permite que o software reconheça uma hierarquia de títulos e subtítulos no documento, já que pessoas cegas não conseguem percebêlas pela diferença do tamanho da fonte ou da cor. Nesse aspecto, este software consegue realizar a leitura de forma contínua e sem interrupções.

Quando se fala em baixa visão, o tipo de fonte utilizada é um fator determinante da acessibilidade digital. Em virtude disso, a REMAT utiliza fontes sem serifa nos artigos, no website e na tipografia do logotipo. $\mathrm{O}$ uso de fontes sem serifa facilita a leitura do usuário. A utilização de fontes com serifa transmite a ideia de algumas letras estarem unidas, devido aos prolongamentos das hastes no final dos caracteres (SALTON et al, 2017). Ainda, Salton et al (2017) ressaltam que é recomendado evitar a utilização de fontes decoradas e cursivas, não só para facilitar a leitura por pessoas com baixa visão, mas também por pessoas com dificuldades de aprendizagem.

Com relação à acessibilidade digital na parte gráfica, a REMAT utiliza a legenda como método de descrever os gráficos ou imagens, além da descrição no decorrer do manuscrito, logo antes ou logo após a figura. Isso possibilita à pessoa cega uma descrição detalhada da parte gráfica, já que os leitores de tela não têm poder para transformar informações sólidas em áudio. Sobre a acessibilidade na parte gráfica, Salton et al (2017) ressaltam que a descrição deste conteúdo pode ser feita de três formas: no parágrafo anterior ou logo após a figura ou gráfico; pela legenda deste objeto; ou utilizando uma caixa de texto alternativo. A caixa de texto alternativo permite escrever uma descrição da figura de forma oculta, sem que apareça visualmente, porém, é interpretada e lida pelo leitor de tela (SALTON et al, 2017). Este último procedimento ainda não é adotado pela REMAT.

Relacionado à acessibilidade na navegação no website, a REMAT utiliza um plugin que permite a alteração do tamanho da fonte em tempo real, auxiliando as pessoas com baixa visão. Para este tipo de deficiência visual, uma melhoria a ser feita no periódico é a disponibilização de recursos para a alteração das configurações de contraste.

Com estas implementações, a REMAT procura tornar acessível tanto os meios de navegação no website quanto o acesso universal e individual dos artigos. 
ANDREIS, G. S. L.; SILVA, L. H. R.; SILVA, R. S.; ARCARO, K.; FUCHS, H. L.; MILHOMENS, D. M.

"Reflexões sobre questões contemporâneas emergentes: acessibilidade digital na remat - revista eletrônica da matemática"

\section{ALCANCE DO PERIÓDICO}

De acordo com os relatórios gerados pelo Sistema Eletrônico de Editoração de Revistas (SEER) utilizado pela REMAT, foram contabilizados 5744 acessos aos resumos e 7772 acessos aos arquivos PDF/A de outubro de 2015 a outubro de 2017, conforme mostra o Quadro 1. A partir dos resultados nota-se uma crescente busca pelos manuscritos publicados no periódico.

Quadro 1 - Número de acessos aos resumos e arquivos PDF/A de 2015 a 2017.

\begin{tabular}{|c|c|c|c|c|c|c|}
\hline \multirow{2}{*}{ Edições } & \multicolumn{15}{|}{$\mathbf{1 5 / 1 0 / \mathbf { 2 0 1 5 } ^ { \mathbf { 6 } } \mathbf { 1 4 / 1 0 / 2 0 1 6 }}$} & $\mathbf{1 5 / 1 0 / 2 0 1 6}$ a 14/10/2017 & \multicolumn{2}{c|}{ Total de acessos } \\
\cline { 2 - 7 } & Resumos & Arquivos & Resumos & Arquivos & Resumos & Arquivos \\
\hline v. 1, n. 1, 2015 & 40 & 21 & 333 & 430 & 373 & $\mathbf{4 5 1}$ \\
\hline v. 1, n. 2, 2015 & 121 & 145 & 901 & 1002 & 1022 & $\mathbf{1 1 4 7}$ \\
\hline v. 2, n. 1, 2016 & 579 & 523 & 804 & 2125 & 1382 & $\mathbf{2 6 4 8}$ \\
\hline v. 2, n. 2, 2016 & - & - & 1841 & 2632 & 1842 & $\mathbf{2 6 3 2}$ \\
\hline v. 3, n. 1, 2017 & - & - & 1125 & 894 & 1125 & $\mathbf{8 9 4}$ \\
\hline Total & $\mathbf{7 4 0}$ & $\mathbf{6 8 9}$ & $\mathbf{5 0 0 4}$ & $\mathbf{7 0 8 3}$ & $\mathbf{5 7 4 4}$ & $\mathbf{7 7 7 2}$ \\
\hline
\end{tabular}

Fonte: Banco de dados estatísticos da REMAT. Dados coletados em 14 de outubro de 2017.

No Quadro 2 apresenta-se os três artigos mais acessados (arquivo PDF/A) bem como a indicação das edições da revista em que foram publicados.

Quadro 2 - Artigos mais acessados em 2015 e 2016.

\begin{tabular}{|l|c|c|}
\hline \multicolumn{1}{|c|}{ Artigo } & Edição & Acessos \\
\hline A Matemática e o lúdico: ensinando frações através de jogos & v. 2, n. 1 & 843 \\
\hline Cálculo de tarifas e tributos incidentes sobre a conta de energia & v. 2, n. 2 & 679 \\
\hline Comparação entre modelos de previsão de demanda: estudo de caso & v. 2, n. 2 & 282 \\
\hline
\end{tabular}

Fonte: Banco de dados estatísticos da REMAT. Dados coletados em 14 de outubro de 2017.

Apesar da REMAT ser uma revista científica apenas com dois anos de circulação, observa-se uma crescente repercussão do periódico. Além do número de acessos aos artigos da REMAT, essa repercussão pode ser evidenciada pela classificação B3 atribuída pela CAPES na área de avaliação Ensino. O gráfico da esquerda da Figura 1 apresenta a distribuição dos 2962 periódicos classificados pela CAPES no quadriênio 2013-2016, na área de avaliação

\footnotetext{
${ }^{6} \mathrm{O}$ número de acessos dos artigos no período de 15/10/2015 à 21/07/2016 foram perdidos pelo sistema.
} 
ANDREIS, G. S. L.; SILVA, L. H. R.; SILVA, R. S.; ARCARO, K.; FUCHS, H. L.; MILHOMENS, D. M. "Reflexões sobre questões contemporâneas emergentes: acessibilidade digital na remat - revista eletrônica da matemática"

Ensino, e o gráfico da direita apresenta a distribuição dos 114 periódicos da área de Matemática, classificados na mesma área de avaliação.

Figura 1 - Extratos dos periódicos classificados pela CAPES no quadriênio 2013-2016, na área de avaliação Ensino: todos os periódicos (esquerda); periódicos da área de Matemática (direita) ${ }^{7}$.
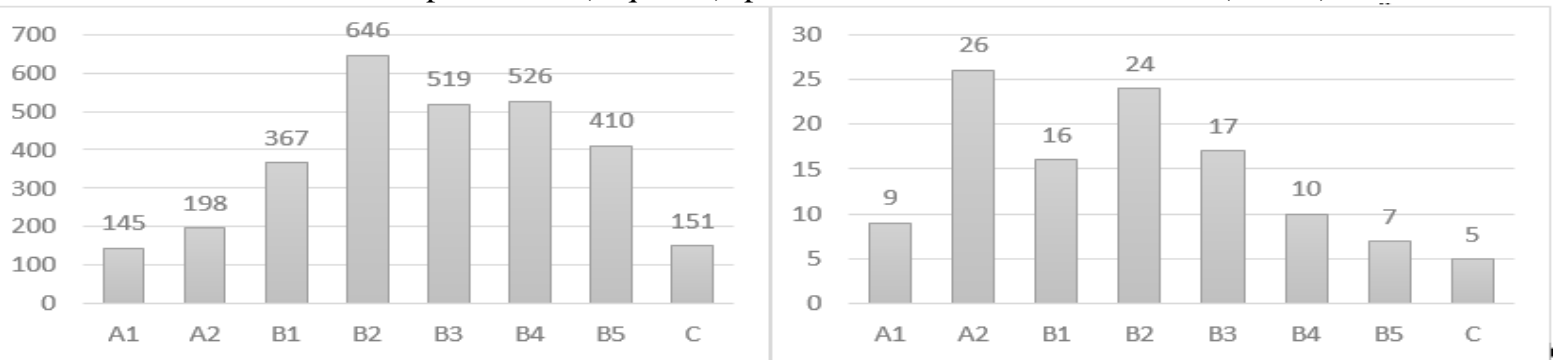

Fonte: Dados coletados no "Qualis Periódicos" da "Plataforma Sucupira” da CAPES , 2017.

O gráfico da esquerda da Figura 2 apresenta a distribuição dos 12 periódicos da área de Matemática criados entre 2013 e 2016 e classificados pela CAPES no quadriênio 2013-2016, na área de avaliação Ensino. O gráfico da direita apresenta a distribuição dos 5 periódicos da área de Matemática criados entre 2015 e 2016, classificados na área de avaliação Ensino.

Figura 2 - Extratos dos periódicos da área de Matemática classificados pela CAPES no quadriênio 2013-2016, na área de avaliação Ensino: criados entre 2013 e 2016 (esquerda); criados entre 2015 e 2016 (direita) ${ }^{9}$.
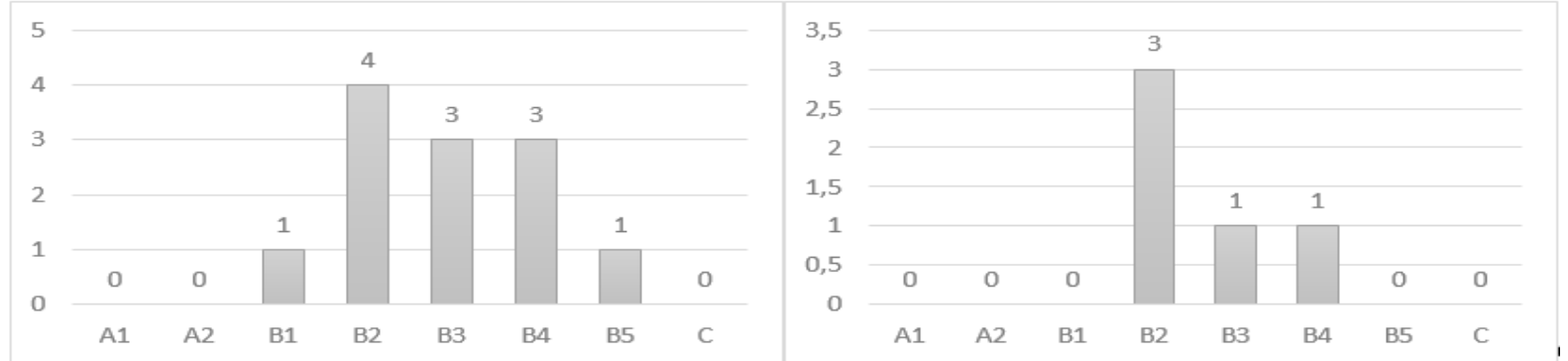

Fonte: Dados coletados no "Qualis Periódicos" da "Plataforma Sucupira” da CAPES $10,2017$.

Com isso, a comunidade de pesquisadores e demais envolvidos no processo de produção e divulgação da REMAT entende que o periódico, além de compartilhar e disseminar conhecimentos científicos, seja também um meio pelo qual novas práticas sobre pensar a Matemática e o ensino da Matemática possam ser veiculadas. Com a revista publicada em um website de acesso livre e irrestrito, torna-se possível alcançar, a nível nacional e internacional, professores, estudantes e demais interessados nas temáticas publicadas. Neste cenário, entendese que a partir da reflexão e ação sobre a demanda contemporânea da acessibilidade digital, o

\footnotetext{
${ }^{7}$ Periódicos com o termo "Matemática" em seu nome, em língua portuguesa ou estrangeira.

${ }^{8}$ Utilizando os filtros: evento de classificação>classificações de periódicos quadriênio 2013-2016; área de avaliação>ensino. Disponível em: <https://sucupira.capes.gov.br/sucupira/public/consultas/coleta/veiculoPublicacaoQualis/listaConsultaGeralPerio dicos.jsf >. Acesso em: 14 out. 2017.

${ }^{9} \mathrm{O}$ ano de criação de cada periódico foi consultado em seu website.

10 Utilizou os filtros: evento de classificação> classificações de periódicos quadriênio 2013-2016; área de avaliação> ensino. Disponível em: <https://sucupira.capes.gov.br/sucupira/public/consultas/coleta/veiculoPublicacaoQualis/listaConsultaGeralPerio dicos.jsf>. Acesso em: 14 out. 2017
} 
ANDREIS, G. S. L.; SILVA, L. H. R.; SILVA, R. S.; ARCARO, K.; FUCHS, H. L.; MILHOMENS, D. M.

"Reflexões sobre questões contemporâneas emergentes: acessibilidade digital na remat - revista eletrônica da matemática"

raio de alcance da revista aumente de medida. A partir dos gráficos apresentados anteriormente nota-se que o produto REMAT, considerado ainda inacabado, deriva do trabalho dos diversos colaboradores que atuam na direção e processo de aperfeiçoamento do periódico.

\section{CONSIDERAÇÕES FINAIS}

A Revista Eletrônica da Matemática, apesar de ser um periódico novo no campo da divulgação científica, tem uma Equipe Editorial consciente e que constantemente reflete e age sobre as demandas contemporâneas emergentes. Entende-se que a acessibilidade digital seja um tema que mobiliza o interesse de diversas áreas do conhecimento, sendo abordada em pesquisas apresentadas em trabalhos de conclusão de cursos de graduação e pós-graduação e também em documentos oficiais. Nesse contexto, nota-se que a visibilidade e o alcance de um periódico demanda que as questões de compartilhamento e socialização do conhecimento mereçam destaque. Tais demandas fazem parte do escopo de trabalho dos editores da REMAT, os quais buscam o aperfeiçoamento dos processos editoriais e na forma em que o material científico é divulgado.

Acredita-se que as ações de aprimoramento realizadas na REMAT sejam consequência do trabalho coletivo, colaborativo, cooperativo e reflexivo do grupo de editores, os quais têm consciência do inacabamento e do caráter evolucionário do processo. Com isso, observa-se que o presente manuscrito tenha alcançado o objetivo de compartilhar junto à comunidade os avanços e o alcance atingidos pela revista, já em um curto espaço de tempo. A primeira avaliação junto à CAPES mostra que a REMAT está em uma rota progressiva, e visa ampliar o espectro de alcance e qualificar ainda mais os processos de divulgação e de compartilhamento do conhecimento científico.

Em suma, e com vistas a projeções futuras, o grupo de colaboradores entende que as questões de acessibilidade digital ainda não foram plenamente alcançadas. Com isso, almeja-se que as ações e implementações futuras na revista sejam: uso de links descritivos, uso de links alternativos e uso das ferramentas de alto contraste. As ações mencionadas anteriormente não esgotam as possibilidades de discussões sobre o assunto, e favorecem e fortalecem o cenário descrito, a fim de que outras reflexões e ações ainda possam acontecer.

\section{AGRADECIMENTOS}


ANDREIS, G. S. L.; SILVA, L. H. R.; SILVA, R. S.; ARCARO, K.; FUCHS, H. L.; MILHOMENS, D. M.

"Reflexões sobre questões contemporâneas emergentes: acessibilidade digital na remat - revista eletrônica da matemática"

Os autores agradecem o apoio financeiro concedido pela Pró-reitoria de Pesquisa, Pósgraduação e Inovação (PROPPI) do IFRS, por meio do Edital 05/2017/PROPPI - Apoio à Edição de Periódicos Científicos do IFRS.

\section{REFERÊNCIAS}

BRASIL. Congresso Nacional. Lei $\mathbf{n}^{0}$ 13.146, de 6 de julho de 2015. Institui a Lei Brasileira de Inclusão da Pessoa com Deficiência (Estatuto da Pessoa com Deficiência). Disponível em: <http://www.planalto.gov.br/ccivil_03/_ato2015-2018/2015/lei/113146.htm>. Acesso em: 12 out. 2017.

SALTON, B. P.; DALL AGNOL, A.; TURCATTI, A. Manual de Acessibilidade em Documentos Digitais. Bento Gonçalves: Instituto Federal de Educação, Ciência e Tecnologia do Rio Grande do Sul, 2017.

SASSAKI, R. K. Inclusão: acessibilidade no lazer, trabalho e educação. Revista Nacional de Reabilitação, São Paulo, ano 11, mar./abr., p. 10-16, 2009. 